\title{
Estimation of Composite Laminate Design Allowables Using the Statistical Characteristics of Lamina Level Test Data
}

\author{
Kyungmin Nam*, Kook Jin Park**, SangJoon Shin*** and Seung Jo Kim**** \\ Department of Aerospace Engineering, Seoul National University, Gwanakro 1, Gwanak-gu, Seoul 08826, Korea
}

\section{Ik-Hyeon Choi ${ }^{* * * * *}$}

Korea Aerospace Research Institute, Gwahak-ro 169-84, Yoosung-gu, Daejeon 34133, Korea

\begin{abstract}
A methodology for determining the design allowables of composite laminates by using lamina level test data and finite element analysis (FEA) is proposed and verified in this paper. An existing method that yields the laminate design allowables by using the complete test results for laminates was improved to reduce the expensive and time-consuming tests. Input property samples for FEA were generated after considering the statistical distribution characteristics of lamina level test data. , and design allowables were derived from several FEA analyses of laminates. To apply and verify the proposed method, Hexcel 8552 IM7 test data were used. For both un-notched and open-hole laminate configurations, it was found that the design allowables obtained from the analysis correctly predicted the laminate test data within the confidence interval. The potential of the present simulation to substitute the laminate tests was demonstrated well.
\end{abstract}

Key words: composite laminate, design allowables, sampling method, estimation of distribution, Monte Carlo simulation

\section{Introduction}

Although composite material has outstanding specific strength and stiffness compared to metallic material, its strength and other properties are not consistent. Thus it requires a significantly larger amount of tests for deducing the design allowables than an isotropic material does.

The A-basis design allowable (A-basis value) is the one which $99 \%$ of the population of material values is expected to equal or exceed, with a $95 \%$ confidence interval. The $B$-basis value is the one which $90 \%$ of the population is expected to equal or exceed.

The building block approach (BBA), which is one of the famous methodologies used in aerospace industries for deducing the properties of composite material, as referred to the composite material handbook $\mathrm{CMH}-17$ [1], specifies the test procedures for the coupon, element, sub-component, and component in the design of a composite material structure.
Coupon test, which consists of lamina level and laminate level tests, requires 20 30 repeated tests to derive statistics for the design allowables. According to the recommendation by the FAA, 1,500 3,000 repeated tests for the lamina level and laminate level tests for the coupon specimen of a composite material will be required. [2]

The laminate level test confirms the influence of the laminate stacking sequence variation and the existence of holes. Since the number of combinations may increase to infinity, only partial subsets will undergo test. On the other hand, since the lamina level test is conducted on unidirectional laminates, most of the required properties can be obtained with a limited number of tests. As such, this paper will seek the possibility of replacing some of the laminate level test with FEA using the lamina level test data.

Many investigations have been reported which apply statistical theory for the uncertainty analysis on a composite material. Astill [3] and Oh [4] applied a Monte Carlo simulation
This is an Open Access article distributed under the terms of the Creative Commons Attribution Non-Commercial License (http://creativecommons.org/licenses/by$\mathrm{nc} / 3.0 /$ which permits unrestricted non-commercial use, distribution, and reproduction in any medium, provided the original work is properly cited.

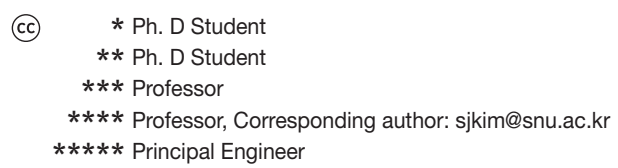


(MCS) to evaluate structural strength, considering the influence of statistical characteristics of composite material properties. Fukuda [5], Goda [6], Yuan [7], and Zhou [8] considered more properties, such as the strength of a fiber or the diameter of a fiber as a random variable that displays either a normal or a Weibull distribution. Vinckenroy [9] used the goodness-of-fit test to estimate the statistical distribution of the material properties such as strength, modulus, Poisson's ratio from the tests, and using that properties with input parameters, performed MCS for the laminate strength.

In the field of reliability, the reliability index is estimated by a variety of methods, such as the first order second moment method (FOSM) and second order second moment method (SOSM) to compute the failure probability. The response surface method (RSM) has been suggested to improve efficiency of the reliability analysis. Lee [10] introduced the MCS , advanced SOFM (ASOFM), and RSM, and compared their efficiencies for analyzing the failure probability and reliability of the composite laminates. With the statistical finite element method (SFEM), statistics was used to introduce variances of input properties into the formulation of the finite element method [11].

Nonetheless, most theories assumed that all the input variables should obey predetermined distributions, mostly either normal or Weibull distribution. However, realistic test data may exhibit a distribution that is not previously known. In extreme situations, the data may not obey or exhibit any existing distributions. From the $\mathrm{CMH}-17$ handbook it can be determineds which type among the existing distributions any specific test data obeys, in order to determine the design allowables for that specific distribution.

In this paper, estimation of the specific distribution will be attempted by accounting for all the single properties provided by test data. This will permit complete examination of the distribution characteristics of each property.

Moreover, the composite material has non-linear characteristics and final failure is reached through degradation after the initial failure. To reflect this, progressive failure analysis (PFA) will be used. For the PFA simulation, an improved version of IPSAP (Internet Parallel Structural Analysis Program) - in-house FEA code which is developed in Seoul National University will be used. This specific program is equipped with a multi-frontal solver and parallel computing algorithm, recently improved and extended [1215]. Park [16] improved it for analyzing composite material characteristics when considering material nonlinearity, continuum damage mechanics, and degradation analysis. He showed that IPSAP predicted un-notched laminate strength within $a \pm 10 \%$ margin of error, especially when compared with tests of Hexcel 8552 IM7 [17].
This paper attempts to present a method to create samples that properly reflect the statistical characteristics of the composite material's test data. It also tries to derive the design allowables from the analytical result, which uses the samples as the input properties. This research is performed under the assumption that the lamina level test results represent the material's properties well. To do this, theories including the pooling method [1], the Anderson-Darling test [18], the Latin hypercube sampling method [19], and rank order correlation [20] will be adopted. A method of estimating $A / B$-basis values from the FEA results will be proposed.

\section{Theoretical Background}

\subsection{Determination of the Design Allowables from Laminate Level Test Data}

To obtain the design allowables used for composite material, both the $99 \%$ lower bound A-basis value and the $90 \%$ lower bound B-basis value at $95 \%$ confidence level are usually estimated by tests, using the building block approach). The $A / B$-basis values of laminates are obtained from the laminate test data as described in Ref. 1-2. . Fig. 1 shows the procedure in a block diagram.

The goodness-of-fit test is then conducted on the pooled data to decide which distribution function best fits the test data. The physical phenomenon such as the property can be modeled with the given data analysis or by the expert's experiences [21]. The property of a material is known to obey either the normal or the Weibull distribution. The normal distribution is selected if there exists no information known in advance [22]. However, this paper does not assume a distribution but conducts the goodness-of-fit test including both normal and Weibull distributions in order to reflect the exact trend of the test data. The goodness-of-fit test includes

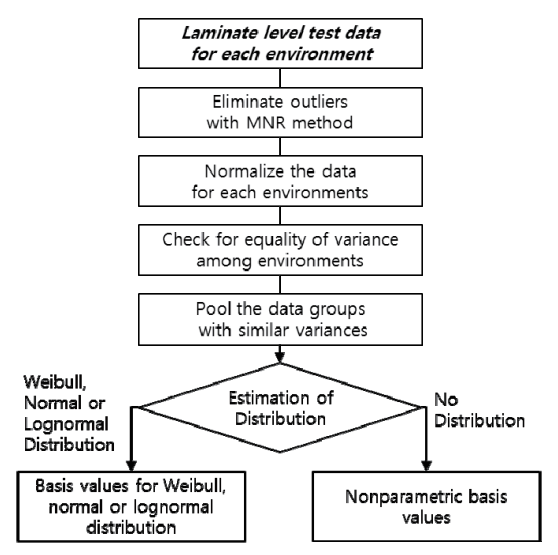

Fig. 1. Procedure of the present method using laminate level test data [1] 
the chi-square test, Kolmogorov-Smirnoff (K-S) test [23], Anderson-Darling (A-D) test, etc.

The A-D test was used in this paper since it is one of the most advanced forms. The A-D test examines for three distributions: Weibull, normal and lognormal distribution. If the test data shows that the $p$-value of a distribution exceeds 0.05 , the test data will be assumed to conform to the distribution. If the Weibull distribution satisfies the condition, the normal and the lognormal distribution are not considered. The lognormal distribution is not considered when $p$-value of the normal distribution exceeds 0.05 . If none of the distributions is satisfied, the basis value will be obtained by the non-parametric method.

$A / B$-basis values are computed by the equations for the selected statistical distribution. The equations for the $A / B$ values are functions of $n$ and coefficients determined by $n$ with the tables in Ref. 1. Confidence in the test data will be determined by the number of the original test data $n$ before pooling. In other words, $A / B$ values are getting larger as the number of test data $n$ increases.

\subsection{Determination of the Design Allowables by Sim- ulation}

The procedure in Section 2.1 is used to estimate the $A / B$-basis values from the laminate test data. To obtain the basis values without laminate test data, the new method is proposed together with the sampling technique and FEA. Only the lamina level test data are used for the proposed method.

The procedure is described in Fig. 2.

Part I: Sample generation from the lamina level test data for FEA

i. Eliminate outliers from the lamina level test data for each environment $(\mathrm{nm}$ test data $\times \mathrm{m}$ properties $\times \mathrm{t}$ environments).

ii. Normalize the lamina level test data for each environment.

iii. Examine for the equality of variance among environmental groups.

iv. Pool the normalized data groups with similar variance characteristics.

v. Perform goodness-of-fit tests for the pooled data.

vi. Sample generation: sampling from the proper distribution function or direct sampling from the test data in accordance with the goodness-of-fit test results.

Steps $\mathrm{i} \sim \mathrm{v}$ above for determining the distribution characteristics of laminate level test data in the original procedure are performed to determine the distribution characteristics of lamina level test data. Since the goodnessof-fit test assumes that the sample population conforms to a specific distribution, it will be rational to extract the sample from the determined distribution function.

Because the $A / B$-basis values are directly extracted from the data if the test sample is deemed not to conform to any specific distribution in the original procedure in Section 2.1, we also extract the samples directly from the test data without making any assumption on distribution. If $N$ samples are selected from each property, a sample set given in $X$ can be obtained.

$$
\begin{aligned}
& X=\left(\begin{array}{c}
x_{1} \\
x_{2} \\
\cdots \\
x_{N}
\end{array}\right) \\
& x_{i}=\left(F_{1}^{t u}, E_{i_{i}}^{t}, v_{12_{i}}^{t}, \cdots, G_{12_{i}}^{s}\right)
\end{aligned}
$$

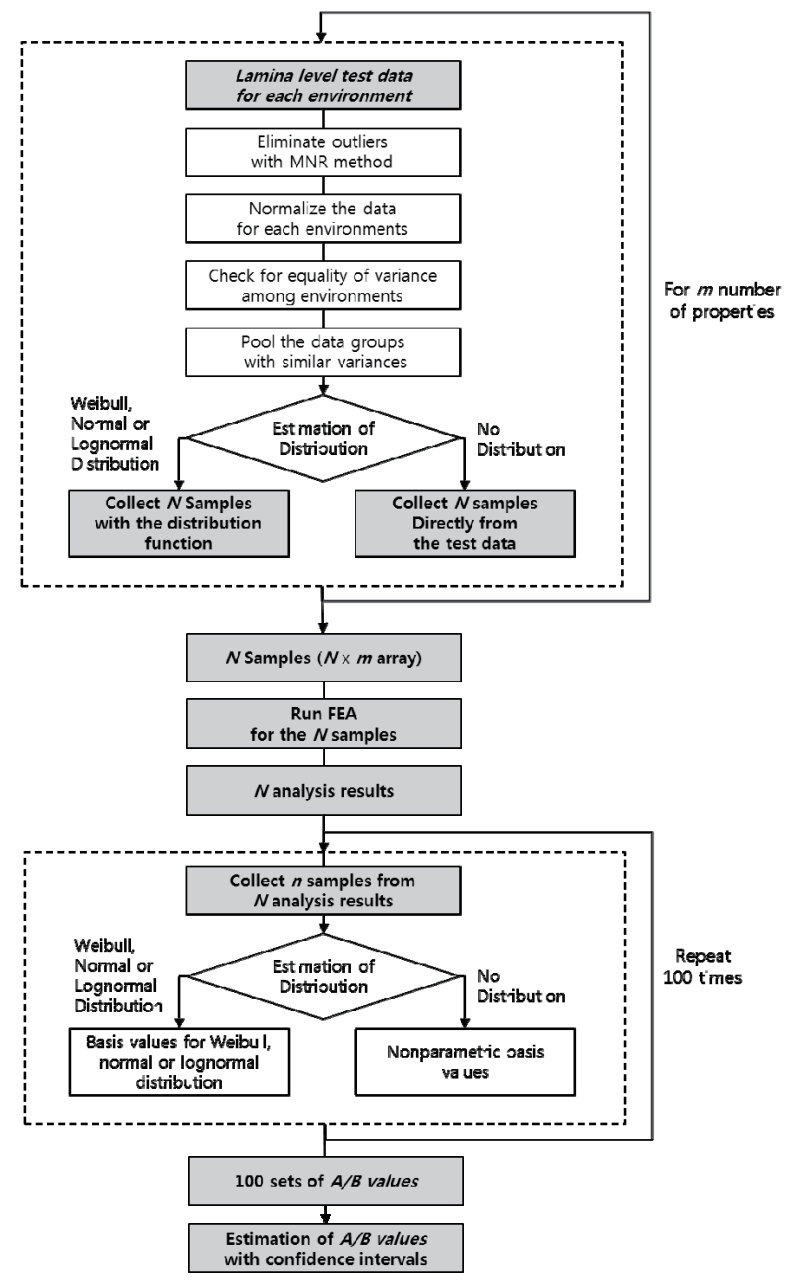

Fig. 2. Procedure of the proposed method using lamina level test data and FEA(shaded blocks are newly added or modified procedures) 
Part II: Calculating A/B-basis values from the FEA results

vii. Calculate laminate strength with FEA for the samples ( $N \times m$ array).

viii. Build subset which consists of random $n_{\min }$ samples from $N$ strength calculation results.

ix. Perform goodness-of-fit test for the subset.

$\mathrm{x}$. Calculate $A / B$-basis values for the proper distribution or with non-parametric methods.

xi. Repeat viii $\sim$ x 100 times.

xii. Estimate $A / B$-basis values with confidence intervals.

Analysis results numbering $N$ can be obtained if $N$ samples are used as input to FEA. The simplest method is to determine the distribution characteristics of such $N$ number of results, and then obtain $k_{A}$ and $k_{B}$ according to $N$ and estimate the $A / B$-basis values. Note, however, that the coefficients for the basis are set to reflect the reliability of the input data as the number of test data increases, and it will not be rational to increase reliability by simply increasing the repetitions of FEA. It is more rational to determine reliability considering the number of lamina level tests used as input.

Estimation of the $A / B$-basis values from $N$ number of analytical results is a point estimation, with a less useful result. It is more useful to present the $A / B$-basis values with a confidence interval, which is a range estimation.

Considering these two factors, Steps viii $\sim$ xii are presented. Assuming the number of the $m$-th property data of the lamina level test to be $n_{m}$, the smallest number is denoted by $n_{\text {min }}$. Conservatively, it is rational to assume the reliability of the input information used for analysis as the level of $n_{\text {min }}$. Subsets having nmin number of data from $N$ number of result values can be extracted, and the $A / B$-basis values are estimated from these subsets. In example, if this process is repeated 100 times randomly, $100 A / B$ values will be obtained. Thus, $95 \%$ or $99 \%$ confidence intervals can be acquired.

\subsection{Monte Carlo Simulation and Latin Hypercube Sampling}

In the analysis of a structure using a composite material with significant uncertainty in its properties, it is difficult to predict behavior accurately with deterministic analysis, which considers only the average values of the properties. Therefore, it is rational to use the statistical approach, which can predict the statistical properties, such as average, distribution, and distribution form.

The main modeling variables such as shape and external force in addition to the properties are given values containing the uncertainties; the techniques reflecting them include the random sampling technique, expansion method, optimization method, statistics-based response function method, and statistics-based approximate integration [11]. Although all those methods (except the random sampling technique) improve efficiency by simplifying the model for approximation, they require additional formulation steps and contain inaccuracies arising from the simplification of the model.

The random sampling method based on the Monte Carlo simulation (MCS) can be applied directly, without modifying the basic model or formula, but requires an increased amount of computations. To supplement the efficiency problem of MCS, sampling techniques such as Latin hypercube sampling, orthogonal array-based sampling, and importance sampling, which all reduces the number of computations required yet obtains the same result, have been developed. They, allow excellent results with a relatively small number of samples [19].

MCS uses the statistical sampling technique to reflect the uncertainty of the model, and executes repeated analyses of the input variable sets extracted from the probability density of input variables of the model, using a random or pseudo-random number. Each input variable set is extracted in accordance with the definition by the probability density function based on the test data or known results. If a sufficient number of samples are used, the input variable distribution can be properly reproduced, and a solution with improved accuracy can be obtained.

Note, however, that such a task will not be suitable for complex models because of the excessive processing time required. To solve this problem, methods such as importance sampling, which assigns different importances to sections, and Latin Hypercube sampling (LHS), which ensures that the samples are uniformly distributed to all sections, have been suggested.

LHS has been applied and improved extensively, since it is simple to apply and highly efficient. Proposed by McKay, et al. [19], it creates samples that are evenly distributed throughout the range, without bias. The space where the input variables are distributed is uniformly divided into the size of sample $N$ so that the samples are extracted in the sample probability in each section. $N$ is generally larger than $k+1$ ( $k$ is the number of changing input variables) and is usually much larger, to ensure accuracy [24]. Random sampling is performed in each divided distribution domain. Only one sampling is allowed in each domain, with sampling performed in all domains. McKay proved that LHS requires fewer samples than basic random sampling method to obtain the same accuracy level.

If there are two or more variables such as $X_{1}, X_{2}, \ldots, X_{d}$, 
each of the independently sampled $X_{1}, X_{2}$, etc., is randomly matched to create sample $X$ composed of $d$ number of samples. In the process, cases of correlation that do not exist originally between the variables being assigned or $d$-dimensional space not being filled may be generated. Such a situation can be improved as the number of samples increases, or removed by checking the correlation between the sampled variables and change ranks of each data with rank order correlation [20].

\section{Numerical Example}

\subsection{Pooling Lamina Level Test Data}

The test data to be used in this paper are of Hexcel 8552 IM7, presented in Ref. 17. The lamina level test consists of five types of tests to obtain the basic properties such as strength and modulus, Poisson's ratio of tension, compression and shear of unidirectional laminates. They are as follows: longitudinal tension (LT) tests, longitudinal compression (LC) tests, transverse tension (TT) tests, transverse compression (LC) tests and in-plane shear (IPS) tests.

Since the property of a composite varies greatly according to temperature and humidity, each test is conducted under the following four temperature and humidity conditions: room temperature dry condition (RTD), cold temperature dry condition (CTD), elevated temperature dry condition (ETD) and elevated temperature wet condition (ETW). As shown in Table 1, the number of data in each environment is 18 20 on average, which is too small to estimate the statistical distribution, particularly for the material. Since the testing of composite material is conducted on a number of cases, it is difficult to obtain a sufficient number of data for each case. The pooling method discussed in $\mathrm{CMH}-17$ [1] is presented to

Table 2. $E_{1}{ }^{t}$ data from lamina level tests [17]

\begin{tabular}{|c|c|c|c|}
\hline \multirow{2}{*}{ Item } & \multicolumn{3}{|c|}{$E_{1}{ }^{t}(\mathrm{Msi})$} \\
\hline & RTD & CTD & ETW \\
\hline \multirow{29}{*}{ Test Data } & 22.553 & 22.425 & 23.285 \\
\hline & 23.43 & 22.078 & 23.975 \\
\hline & 22.588 & 22.573 & 23.793 \\
\hline & 23.785 & 22.937 & 23.382 \\
\hline & 23.433 & 21.852 & 25.578 \\
\hline & 23.528 & 22.667 & 23.761 \\
\hline & 22.795 & 22.342 & 24.389 \\
\hline & 23.305 & 22.541 & 23.281 \\
\hline & 23.076 & 22.54 & 23.977 \\
\hline & 21.835 & 22.436 & 23.622 \\
\hline & 23.716 & 22.801 & 24.702 \\
\hline & 23.731 & 23.099 & 23.496 \\
\hline & 20.707 & 22.103 & 23.719 \\
\hline & 23.225 & 22.034 & 23.828 \\
\hline & 23.053 & 23.03 & 23.558 \\
\hline & 22.09 & 22.039 & 23.738 \\
\hline & 22.973 & 22.651 & 24.063 \\
\hline & 23.941 & 23.119 & 24.356 \\
\hline & & 23.219 & 23.222 \\
\hline & & 22.468 & 24.329 \\
\hline & & 22.832 & 24.378 \\
\hline & & 22.706 & 23.449 \\
\hline & & & 24.831 \\
\hline & & & 23.769 \\
\hline & & & 23.93 \\
\hline & & & 24.023 \\
\hline & & & 24.79 \\
\hline & & & 24.023 \\
\hline & & & 24.778 \\
\hline Average & 22.987 & 22.567 & 24.001 \\
\hline Std. Dev. & 0.812 & 0.387 & 0.557 \\
\hline \#of Data $(n)$ & 18 & 22 & 29 \\
\hline
\end{tabular}

Table 1. Number of test data for Hexcel IM7 lamina level properties [17]

\begin{tabular}{|c|c|c|c|c|c|c|c|}
\hline \multirow{2}{*}{ Property } & \multirow{2}{*}{ Symbol } & \multicolumn{4}{|c|}{ \# of Data } & \multirow{2}{*}{ Test } & \multirow{2}{*}{$\begin{array}{l}\text { Stacking } \\
\text { sequence }\end{array}$} \\
\hline & & RTD & CTD & ETD & ETW & & \\
\hline LT strength & $F_{1}^{t u}$ & 18 & 22 & - & 18 & \multirow{3}{*}{$\begin{array}{c}\text { Longitudinal } \\
\text { Tension }\end{array}$} & \multirow{3}{*}[0]{$_{6}$} \\
\hline LT modulus & $E_{l}^{t}$ & 18 & 22 & - & 29 & & \\
\hline $\begin{array}{l}\text { LT Poisson's } \\
\text { ratio }\end{array}$ & $\nu_{12}^{t}$ & 18 & 22 & - & 25 & & \\
\hline TT strength & $F_{2}^{t u}$ & 20 & 21 & - & 19 & \multirow{2}{*}{$\begin{array}{c}\text { Transverse } \\
\text { Tension }\end{array}$} & \multirow{2}{*}[90]{$_{11}$} \\
\hline TT modulus & $E_{2}{ }^{t}$ & 20 & 21 & - & 19 & & \\
\hline LC modulus & $E_{l}{ }^{c}$ & 15 & 20 & 17 & 35 & \multirow{2}{*}{$\begin{array}{l}\text { Longitudinal } \\
\text { Compression }\end{array}$} & \multirow[b]{2}{*}[0]{$_{14}$} \\
\hline $\begin{array}{l}\text { LC Poisson's } \\
\text { ratio }\end{array}$ & $\nu_{12}^{c}$ & 15 & 20 & 17 & 35 & & \\
\hline TC strength & $F_{2}^{c u}$ & 20 & 20 & - & 25 & \multirow{3}{*}{$\begin{array}{l}\text { Transverse } \\
\text { Compression }\end{array}$} & \multirow{3}{*}[90]{$_{14}$} \\
\hline TC modulus & $E_{2}^{c}$ & 20 & 20 & - & 9 & & \\
\hline $\begin{array}{l}\text { TC Poisson's } \\
\text { ratio }\end{array}$ & $\nu_{21}{ }^{c}$ & 20 & 20 & - & 9 & & \\
\hline $\begin{array}{c}\text { IPS } 0.2 \% \\
\text { yield strength }\end{array}$ & $F_{12}{ }^{s 0.2 \% \varepsilon}$ & 12 & - & - & 19 & \multirow{3}{*}{$\begin{array}{l}\text { In-Plane } \\
\text { Shear }\end{array}$} & \multirow{3}{*}{$\begin{array}{l}{[+45 /} \\
-45]_{3 \mathrm{~s}}\end{array}$} \\
\hline IPS strength & $F_{12}{ }^{s 5 \% \varepsilon}$ & 16 & 21 & - & 20 & & \\
\hline IPS modulus & $G_{12}{ }^{s}$ & 16 & 21 & - & 20 & & \\
\hline
\end{tabular}


overcome such difficulties.

This section describes the pooling procedure with the $E_{1}{ }^{t}$ property, which is the longitudinal tensile modulus of the material. The raw data for each environment of $E_{1}{ }^{t}$ are given in Table 2. Firstly, outlier removal and normalizing for each environment's data are performed in accordance with the procedure explained in Section 2. The normalized data are shown in Table 3. The variance analysis of the sets of all the environmental groups yielded a $p$-value of 0.34 , indicating similar variance characteristics. Therefore, the data of all three environments of $E_{l}^{t}$ can be grouped together to estimate the distribution characteristics of the lamina level $E_{1}{ }^{t}$ property. As a result, the data size became 68; this was sufficient to infer the distribution.

\subsection{Estimation of Distribution and Sampling}

The consistency of the results with the normal, Weibull, and lognormal distributions was evaluated by using the Anderson-Darling goodness-of-fit test [18]. Table 4 shows

Table 3. Normalized data of $E_{1}^{t}$

\begin{tabular}{|c|c|c|c|}
\hline \multirow{2}{*}{ Item } & \multicolumn{3}{|c|}{$E_{l}{ }^{t}(\mathrm{Msi})$} \\
\hline & RTD & CTD & ETW \\
\hline \multirow{29}{*}{$\begin{array}{l}\text { Normalized } \\
\text { Data }\end{array}$} & 0.9754 & 0.9937 & 0.9702 \\
\hline & 1.0134 & 0.9783 & 0.9989 \\
\hline & 0.9769 & 1.0002 & 0.9913 \\
\hline & 1.0287 & 1.0164 & 0.9742 \\
\hline & 1.0135 & 0.9683 & 1.0657 \\
\hline & 1.0176 & 1.0044 & 0.9900 \\
\hline & 0.9859 & 0.9900 & 1.0162 \\
\hline & 1.0080 & 0.9988 & 0.9700 \\
\hline & 0.9981 & 0.9988 & 0.9990 \\
\hline & 0.9444 & 0.9942 & 0.9842 \\
\hline & 1.0257 & 1.0103 & 1.0292 \\
\hline & 1.0264 & 1.0235 & 0.9790 \\
\hline & 1.0045 & 0.9794 & 0.9883 \\
\hline & 0.9971 & 0.9763 & 0.9928 \\
\hline & 0.9554 & 1.0205 & 0.9815 \\
\hline & 0.9936 & 0.9766 & 0.9890 \\
\hline & 1.0355 & 1.0037 & 1.0026 \\
\hline & & 1.0244 & 1.0148 \\
\hline & & 1.0289 & 0.9675 \\
\hline & & 0.9956 & 1.0137 \\
\hline & & 1.0117 & 1.0157 \\
\hline & & 1.0061 & 0.9770 \\
\hline & & & 1.0346 \\
\hline & & & 0.9903 \\
\hline & & & 0.9970 \\
\hline & & & 1.0009 \\
\hline & & & 1.0329 \\
\hline & & & 1.0009 \\
\hline & & & 1.0324 \\
\hline Average & 1.0000 & 1.0000 & 1.0000 \\
\hline Std. Dev. & 0.0258 & 0.0172 & 0.0232 \\
\hline \#of $\operatorname{Data}(n)$ & 17 & 22 & 29 \\
\hline
\end{tabular}

the result. The normal fit of initial data and pooled data are shown in Figs. 3 and 4.

As a result of the procedure described in this Section 2.1 , the population of $E_{1}^{t}$ can be considered to have the normal distribution, and sampling can be performed using the normal probability distribution function. By the same procedure, the other properties of IM7 were pooled, and the resulting of estimation of distribution characteristics is given in Table 5.

Sampling is performed in accordance with the result of the estimation of distribution. Properties that follow the distributions are sampled using probability distribution functions and a random number generator. Since general random number generators give random numbers in $[0,1]$ for uniform distribution, to obtain random numbers that are not uniformly distributed, an inverse transform

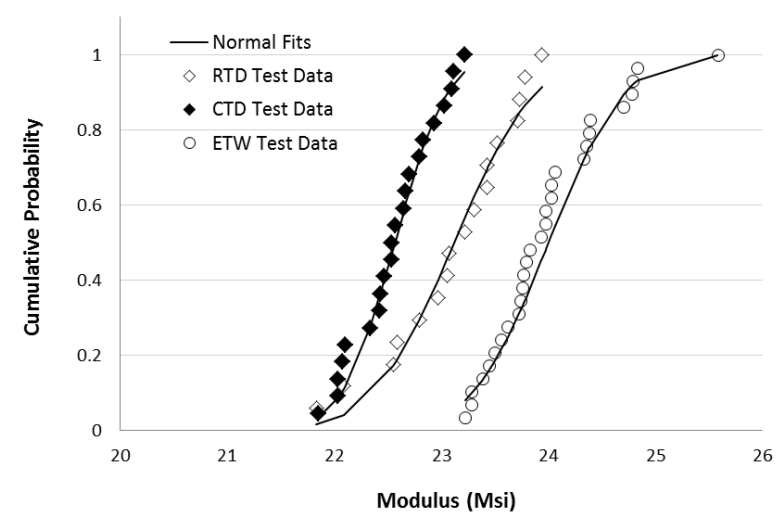

Fig. 3. Normal fit of test data for each environment

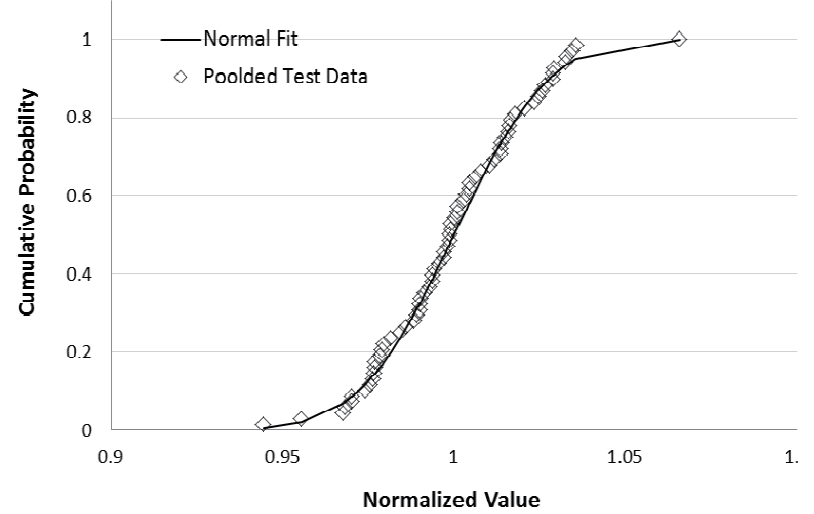

Fig. 4. Normal fit of pooled data

Table 4. Anderson-Darling goodness-of-fit tests for $E_{1}{ }^{t}$

\begin{tabular}{ccc}
\hline Distribution & $p$-value of A-D test & Result \\
\hline Weibull & 0.005 & rejected $(p<0.05)$ \\
Normal & 0.786 & accepted $(p>0.05)$ \\
Lognormal & 0.815 & not considered \\
\hline
\end{tabular}


method is generally used [25]. Because the Weibull distribution function can be transformed to an inverse function, random numbers for this distribution can be obtained by inverse transform method. But there is no inverse function for normal and lognormal distributions;

Table 5. Distribution estimation of pooled data of lamina level properties

\begin{tabular}{|c|c|c|c|c|c|c|}
\hline \multirow{2}{*}{ Property } & \multicolumn{5}{|c|}{ Sample Sets } & \multirow{2}{*}{$\begin{array}{l}\text { Sampling } \\
\text { Method }\end{array}$} \\
\hline & $X_{I}$ & $X_{2}$ & $X_{3}$ & $\ldots$ & $X_{l, 000}$ & \\
\hline$F_{l}^{t u}$ & 359.19 & 393.75 & 388.35 & $\ldots$ & 379.48 & Normal Dist. \\
\hline$E_{l}^{t}$ & 23.392 & 23.226 & 23.181 & & 23.786 & Normal Dist. \\
\hline$\nu_{12}{ }^{t}$ & 0.3150 & 0.3080 & 0.3060 & $\ldots$ & 0.3190 & Direct Sampling \\
\hline$F_{2}^{t u}$ & 8.3573 & 9.4621 & 8.6380 & & 9.8765 & Weibull Dist. \\
\hline$E_{2}^{t}$ & 1.4010 & 1.3330 & 1.2973 & $\ldots$ & 1.3070 & Direct Sampling \\
\hline$E_{l}^{c}$ & 18.341 & 19.827 & 23.517 & & 18.137 & Normal Dist. \\
\hline$\nu_{12}^{c}$ & 0.3261 & 0.3458 & 0.3446 & & 0.3394 & Lognormal Dist. \\
\hline$F_{2}^{c u}$ & 46.486 & 42.616 & 39.043 & $\ldots$ & 42.841 & Weibull Dist. \\
\hline$E_{2}^{c}$ & 1.4019 & 1.4288 & 1.2817 & & 1.3139 & Normal Dist. \\
\hline$\nu_{21}^{c}$ & 0.0245 & 0.0247 & 0.0228 & $\ldots$ & 0.0208 & Weibull Dist. \\
\hline$F_{12}{ }^{50.2 \% 8}$ & 13.616 & 12.948 & 13.072 & & 12.713 & Weibull Dist. \\
\hline$F_{12}{ }^{s 5 \%}$ & 8.1170 & 7.8693 & 8.2790 & & 8.2790 & Direct Sampling \\
\hline$G_{12}{ }^{s}$ & 0.6887 & 0.7101 & 0.6700 & $\ldots$ & 0.6637 & Normal Dist. \\
\hline
\end{tabular}

we used an approximate inverse transform method [26]. Properties not obeying those three distributions are sampled directly in the pooled test data. The structure of the present sample set is shown in Table 6, when the sample set size is 1,000 .

\subsection{Computation of the Design Allowable from Sim- ulation Results}

Since lamina level test data for the sampling have 12 25 data for each case, only the B-basis value can be obtained [1]. Un-notched and open-hole laminate configurations for

Table 7. Laminate configurations for simulation

\begin{tabular}{ccc}
\hline \multicolumn{1}{c}{ Laminate } & & Stacking Sequence \\
\hline \multirow{2}{*}{$\begin{array}{c}\text { Un-Notched laminate } \\
\text { Tension }\end{array}$} & UNT1 & {$[45,0,-45,90]_{2 \mathrm{~s}}$} \\
& UNT2 & {$[45,-45,0,45,-45,90,45,-45,45,-45]_{\mathrm{s}}$} \\
& UNT3 & {$[0,45,0,90,0,-45,0,45,0,-45]_{\mathrm{s}}$} \\
\hline \multirow{2}{*}{$\begin{array}{c}\text { Open-Hole laminate } \\
\text { Tension }\end{array}$} & OHT1 & {$[45,0,-45,90]_{2 \mathrm{~s}}$} \\
& OHT2 & {$[45,-45,0,45,-45,90,45,-45,45,-45]_{\mathrm{s}}$} \\
& OHT3 & {$[0,45,0,90,0,-45,0,45,0,-45]_{\mathrm{s}}$} \\
\hline
\end{tabular}

Table 6. Sample set of 1,000 samples for analysis

\begin{tabular}{ccccccc}
\hline Property & \multicolumn{5}{c}{ Sample Sets } & \multicolumn{2}{c}{ Sampling } \\
& $X_{I}$ & $X_{2}$ & $X_{3}$ & $\ldots$ & $X_{1,000}$ & Method \\
\hline$F_{1}{ }^{t}{ }^{t}$ & 359.19 & 393.75 & 388.35 & $\ldots$ & 379.48 & Normal Dist. \\
$E_{1}{ }^{t}$ & 23.392 & 23.226 & 23.181 & $\ldots$ & 23.786 & Normal Dist. \\
$\nu_{12}{ }^{t}$ & 0.3150 & 0.3080 & 0.3060 & $\ldots$ & 0.3190 & Direct Sampling \\
$F_{2}{ }^{t u}$ & 8.3573 & 9.4621 & 8.6380 & $\ldots$ & 9.8765 & Weibull Dist. \\
$E_{2}{ }^{t}$ & 1.4010 & 1.3330 & 1.2973 & $\ldots$ & 1.3070 & Direct Sampling \\
$E_{1}{ }^{c}$ & 18.341 & 19.827 & 23.517 & $\ldots$ & 18.137 & Normal Dist. \\
$\nu_{12}{ }^{c}$ & 0.3261 & 0.3458 & 0.3446 & $\ldots$ & 0.3394 & Lognormal Dist. \\
$F_{2}{ }^{c u}$ & 46.486 & 42.616 & 39.043 & $\ldots$ & 42.841 & Weibull Dist. \\
$E_{2}{ }^{c}$ & 1.4019 & 1.4288 & 1.2817 & $\ldots$ & 1.3139 & Normal Dist. \\
$\nu_{21}{ }^{c}$ & 0.0245 & 0.0247 & 0.0228 & $\ldots$ & 0.0208 & Weibull Dist. \\
$F_{12}{ }^{s 0.2 \% \varepsilon}$ & 13.616 & 12.948 & 13.072 & $\ldots$ & 12.713 & Weibull Dist. \\
$F_{12}{ }^{s 5 \% \varepsilon}$ & 8.1170 & 7.8693 & 8.2790 & $\ldots$ & 8.2790 & Direct Sampling \\
$G_{12}{ }^{s}$ & 0.6887 & 0.7101 & 0.6700 & $\ldots$ & 0.6637 & Normal Dist. \\
\hline
\end{tabular}

Table 8. Laminate tensile strength analysis results in simulation

\begin{tabular}{ccccccc}
\hline \multirow{2}{*}{ Input Sample Set } & \multicolumn{7}{c}{ Tensile Strength Analysis Results (ksi) } \\
& UNT1 & UNT2 & UNT3 & OHT1 & OHT2 & OHT3 \\
\hline$X_{1}$ & 100.29 & 66.954 & 161.03 & 59.206 & 46.187 & 87.125 \\
$X_{2}$ & 109.84 & 71.971 & 176.50 & 62.362 & 47.377 & 92.066 \\
$X_{3}$ & 109.94 & 72.016 & 174.91 & 63.446 & 47.936 & 92.969 \\
$X_{4}$ & 99.490 & 66.297 & 160.92 & 57.993 & 44.244 & 85.558 \\
$X_{5}$ & 102.17 & 67.825 & 163.07 & 60.768 & 47.467 & 89.599 \\
$\ldots$ & $\ldots$ & $\ldots$ & $\ldots$ & $\ldots$ & $\ldots$ & $\ldots$ \\
$X_{\text {I000 }}$ & 104.85 & 69.253 & 167.16 & 61.570 & 47.392 & 90.992 \\
\hline Sample Mean & 101.79 & 67.566 & 163.45 & 59.835 & 46.096 & 88.542 \\
Sample Std. Dev. & 4.759 & 2.675 & 6.950 & 2.018 & 1.349 & 2.779 \\
\hline
\end{tabular}


this analysis are presented in Table 7. Longitudinal tensile strength analysis results on the un-notched and open-hole laminates under tensile loading using the sampling method (Section 2.2) and PFA FEA are given in Table 8.

For OHT1, the methodology for determining the $B$-basis value is presented as follows. First, the AndersonDarling goodness-of-fit test was performed to estimate the distribution characteristic of 1,000 strength values for OHT1. As shown in Table 9, OHT1 tensile strength obeys the normal distribution.

Among the lamina level test data used for the sample generation, the smallest number of tests $n_{\min }$ is 12 , for the property $F_{12}{ }^{55 \% \varepsilon}$ of in-plane shear tests. Now, a subset of 12 values is collected randomly as shown in Table 10. For the normal distribution, $k_{B}=2.211$ can be found in Table 8.5.14 in Ref. 1 for $n=12$. With following equation, the $B$-basis value for the subset is obtained.

$$
\mathrm{B}=\bar{x}-k_{B} S=60.051-2.211 \times 2.114=55.377
$$

For 100 subsets collected randomly, 100 B-basis values are obtained. For those 100 values, we can find both the lowest and highest discrepancy levels for a $99 \%$ confidence

Table 9. Anderson-Darling goodness-of-fit tests for the simulation results

\begin{tabular}{ccc}
\hline Distribution & $p$-value of A-D test & Result \\
\hline Weibull & 0 & rejected $(p<0.05)$ \\
Normal & 0.093 & accepted $(p>0.05)$ \\
Lognormal & 0.526 & not considered \\
\hline
\end{tabular}

Table 10. Subset for B-basis value computation

\begin{tabular}{cc}
\hline $\begin{array}{c}\text { \# in the Original 1,000 Sets } \\
\text { (random) }\end{array}$ & Tensile Strength of OHT1 (ksi) \\
\hline 72 & 63.360 \\
678 & 57.538 \\
641 & 61.611 \\
883 & 57.533 \\
313 & 61.641 \\
591 & 61.686 \\
870 & 61.157 \\
290 & 59.084 \\
433 & 62.935 \\
993 & 60.548 \\
142 & 62.742 \\
350 & 57.981 \\
\hline Subset Mean & 60.051 \\
Subset Std. Dev. & 2.114 \\
\hline B-Basis Value & 55.377 \\
\hline
\end{tabular}

interval, as shown in Table 11. The final result of the $B$-basis value for OHT1 laminate strength is presented as follows.

$$
\mathrm{B}=54.697 \pm 2.687(\mathrm{ksi}) \quad(99 \% \text { confidence interval })
$$

For the other laminate configurations (UNT1/2/3 and OHT2/3), the B-basis values are calculated by the same process. Table 12 and Fig. 5 show the analysis results. Overall, the analysis results for the B-basis values predict the test result well in the discrepancy range.

Table 11. B-basis values for OHT1 laminate

\begin{tabular}{cc}
\hline Subset \# & $\begin{array}{c}\text { OHT1 Strength } \\
\text { B-Basis Value (ksi) }\end{array}$ \\
\hline 1 & 55.377 \\
2 & 55.356 \\
3 & 54.218 \\
4 & 53.486 \\
5 & 57.277 \\
$\ldots$ & $\ldots$ \\
100 & 54.883 \\
\hline $0.5 \%$ Value & 52.010 \\
$99.5 \%$ Value & 57.385
\end{tabular}

Table 12. B-basis value computation results

\begin{tabular}{ccc}
\hline \multirow{2}{*}{ Laminate Model } & \multicolumn{2}{c}{ B-Basis Value (ksi) } \\
& Analysis & Test \\
\hline UNT1 & $90.927 \pm 6.667$ & 92.427 \\
UNT2 & $59.454 \pm 5.652$ & 58.718 \\
UNT3 & $145.350 \pm 12.704$ & 156.435 \\
\hline OHT1 & $54.697 \pm 2.687$ & 54.459 \\
OHT2 & $42.358 \pm 2.524$ & 41.292 \\
OHT3 & $81.071 \pm 4.746$ & 77.869 \\
\hline
\end{tabular}

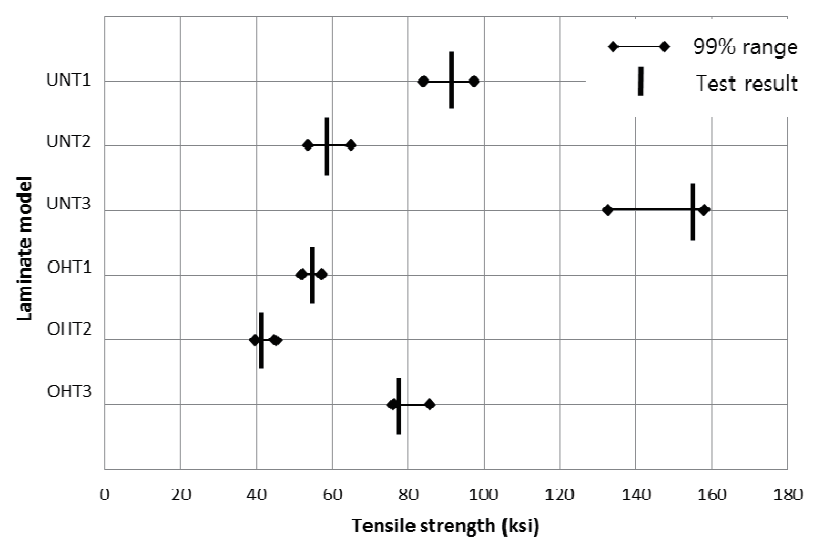

Fig. 5. B-basis value estimations with $99 \%$ confidence interval 


\section{Concluding Remarks}

In this paper, a methodology is proposed to predict the design allowables for laminates, without the costly and timeconsuming laminate level tests, through a simulation using lamina level test data.

To accurately estimate distribution characteristics of the properties from lamina level test data, a pooling method and Anderson-Darling goodness-of-fit test were conducted. The Latin hypercube sampling method was used to create welldistributed random samples.

By using the samples which have the test results' distribution characteristics for each property and progressive failure analysis by an improved version of IPSAP was performed to obtain the tensile strengths for the three un-notched laminates and the three open-hole laminate configurations. By collecting subsets in the strength analysis results, the B-basis values were obtained with certain discrepancy range. The $B$-basis values from laminate level tests were predicted well with the analysis, and the potential of substituting simulation for laminate tests was well demonstrated.

This methodology was performed under the assumption that the lamina level test data reflects statistically enough the lamina population's uncertainty characteristics from batches, environments, voids and defects. If the number of the test data is too small or only partly reflects the population characteristics, the analysis results cannot be generalized to obtain design allowables. To complement this, the pooling method was used. Confidence of the analysis result can be improved by using large number of data that influences the uncertainty property better.

When determining the parameters for the basis computation, the reliability level was assumed in accordance with the smallest number of data among the properties of the lamina level test. But, for the properties contributing to the result which have different sensitivities, more study is needed for determining the reliability level, considering the number of data of the property of higher sensitivity.

\section{Acknowledgement}

This study was supported by the Korean Ministry of Land, Infrastructure and Transport Affairs (MOLIT) through the Air Transportation Advancement Program (ATAP) of the Korea Agency for Infrastructure Technology Advancement (KAIA).

\section{References}

[1] CMH-17, Composite Materials Handbook, Federal Aviation Administration, 2012.

[2] DOT/FAA/AR-03/19, Material Qualification and Equivalency for Polymer Matrix Composite Material Systems: Updated Procedure, Federal Aviation Administration, 2003.

[3] J. Astill, C. Nosseir, and M. Shinozuka, "Impact Loading on Structures with Random Properties", J. Structural Mechanics, Vol. 1, No. 1, 1972, pp. 63-77.

[4] K. P. Oh, "A Monte Carlo Study of the Strength of Unidirectional Fiber-Reinforced Composites", J. Composite Materials, Vol. 13, 1979, pp. 311-328.

[5] H. Fukuda, and T. W. Chou, "Monte Carlo Simulation of the Strength of Hybrid Composites", J. Composite Materials, Vol. 16, 1982, pp. 371-385.

[6] K. Goda, and S. L. Pheonix, "Reliability Approach to the Tensile Strength of Unidirectional CFRP Composites by Monte Carlo Simulation in a Shear-Lag Model", Composite Science and Technology, Vol. 50, 1994, pp. 457-468.

[7] J. Yuan, Y. Xia, and B. Yang, "A Note on the Monte Carlo Simulation of the Tensile Deformation and Failure Process of Unidirectional Composites", Composite Science and Technology, Vol. 52, 1994, pp. 197-204.

[8]Y.Zhou, H. Mahfuz, and S. Jeelani, "NumericalSimulation for High Strain Rate Failure Process of Unidirectional Sicf-Al Composites", Int. J. Damage Mechanics, Vol. 14, No. 4, 2005, pp. 321-341.

[9] G. Vinckenroy, and W. Wilde, "The Use of Monte Carlo Techniques in Statistical Finite Element Methods for the Determination of the Structural Behaviour of Composite Materials Structural Components", Composite Structures, Vol. 32, 1995, pp. 247-253.

[10] S. J. Lee, I. G. Kim, and M. H. Jang, "Reliability Analysis for Composite Plate with the Various Design Requirements", J. The Korean Society for Composite Materials, Vol. 20, No. 4 , 2007, pp. 25-30.

[11] P. Marek, M. Gustar, and T. Anagnos, SimulationBased Reliability Assessment, CRC Press, 1995.

[12] J. H. Kim, and S. J. Kim, "A Multifrontal Solver Combined with Graph Partitioners", AIAA Journal, Vol. 38 No. 8, Aug. 1999, pp. 964-970.

[13] S. J. Kim, C. S. Lee, and J. H. Kim, "Large-Scale Structural Analysis by Parallel Multifrontal Solver through Internet Based Pcs", AIAA Journal, Vol. 40, No. 2, 2002, pp. 359-367.

[14] J. H. Kim, C. S. Lee, and S. J. Kim, "High-Performance Domain-Wise Parallel Direct Solver for Large-Scale Structural Analysis", AIAA Journal, Vol. 43, No. 3, 2005, pp. 
662-67034.

[15] J. W. Park, S. H. Park, and S. J. Kim, “Optimization with High-Cost Objective Function Evaluations in a Computing Grid and an Application to Simulation-Based Design", Int. J. High Performance Computing Applications, Vol. 23, No. 1, 2009, pp. 62-83.

[16] K. J. Park, H. Kang et al., "Strength Prediction on Composite Laminates Including Material Nonlinearity and Continuum Damage Mechanics", Korean J. of Aerospace Engineering, Vol. 42, No. 11, 2014, pp. 927-936.

[17] K. Marlett, et al., Hexcel 8552 IM7 Unidirectional Prepreg 190 gsm \& 35\%RC Qualification Material Property Data Report, CAM-RP-2009-015 Rev. A, NIAR, Wichita State University, 2011.

[18] T. W. Anderson, and D. A. Darling, "Asymptotic Theory of Certain "Goodness of Fit" Criteria Based on Stochastic Processes", The Annals of Mathematical Statistics, Vol. 23, No. 2, 1952, pp. 193-212.

[19] M. D. McKay, R. J. Beckman, and W. J. Conover, "A Comparison of Three Methods for Selection Values of Input Variables in the Analysis of Output from a Computer Code",
Technometrics, Vol. 21, No. 2, 1979, pp. 239-245.

[20] R. L. Iman, and W. J. Conover, "A Distribution-Free Approach to Inducing Rank Correlation among Input Variables", Communications in Statistics-Simulation and Computation, Vol. 11, 1982, pp. 311-334.

[21] D. Vose, Risk Analysis: A Quantitative Guide, John Wiley \& Sons, New York, 2000

[22] S. Marino, et al., "A Methodology for Performing Global Uncertainty and Sensitivity Analysis in Systems Biology", J. Theoretical Biology, Vol. 254, No. 1, 2008, pp. 178-196.

[23] Frank J. Massey Jr., "The Kolmogorov-Smirnov Test for Goodness of Fit", J. the American Statistical Association, Vol. 46, No. 253, 1951, pp. 68-78.

[24] M. L. Shooman, Probabilistic Reliability: An Engineering Approach, McGraw-Hill, 1968.

[25] A. Haldar, and S. Mahadevan, Reliability Assessment Using Stochastic Finite Element Analysis, John Wiley and Sons, 2000.

[26] R. Billinton, and W. Li, Reliability Assessment of Electric Power Systems Using Monte Carlo Methods, Plenum Press, 1994. 